\title{
Inactivation of Viable Surrogates for the Select Agents Virulent Newcastle Disease Virus and Highly Pathogenic Avian Influenza Virus Using Either Commercial Lysis Buffer or Heat
}

Applied Biosafety:

Journal of ABSA International 2019, Vol. 24(4) 189-199

(C) ABSA International 2019

Article reuse guidelines:

sagepub.com/journals-permissions DOI: $10.1177 / 1535676019888920$ journals.sagepub.com/home/apb (QSAGE

\author{
Katrina Alger' $\odot$, Hon Ip', Jeffrey Hall', Sean Nashold', \\ Katherine Richgels', and Carrie Smith'
}

\begin{abstract}
Introduction: Federal Select Agent Program regulations require laboratories to document a validated procedure for inactivating select agents prior to movement outside registered space. Avian influenza viruses and virulent Newcastle disease virus (vNDV) are cultured in chicken amnio-allantoic fluid (AAF), but the efficacy of commercial lysis buffers to inactivate viruses in protein-rich media has not been documented.

Objectives: We assesses the efficacy of MagMAX'M lysis buffer for inactivating highly pathogenic avian influenza virus (HPAIV) and VNDV in chicken AAF and confirm the inactivation of avian influenza in serum using heat.

Methods: Low pathogenic avian influenza virus (LPAIV) and avian paramyxovirus subtype-I (APMV-I) were incubated with lysis buffer and tested for viability. Known viable LPAIV and APMV-I RNA was extracted from AAF using MagMAX'M_-96 AI/ND Viral RNA Isolation kit, and the eluate was tested for remaining infectious agent. Finally, inactivation of LPAIV in serum was examined over 3 combinations of temperature and incubation time.

Results: MagMAX'M lysis buffer inactivated both LPAIV and APMV-I in AAF when incubated for 30 minutes at room temperature. The full extraction process eliminated viable virus from the final RNA eluate. LPAIV in serum heated to $70^{\circ} \mathrm{C}$ for 30 minutes was rendered noninfectious.

Conclusion: The ability of a diagnostic laboratory to move samples from one space to another is critical to maintaining biosecurity as well as efficient laboratory workflow. Our study demonstrates a method to ensure the inactivation of viable avian influenza and avian paramyxoviruses in AAF, RNA eluate, and viable avian influenza virus in sera.
\end{abstract}

\section{Keywords}

biological select agents and toxins, biorisk management, Federal Select Agent Program, pathogen, infectious agent, BSL-3

\section{Introduction}

The Federal Select Agent Program (FSAP), managed jointly by the Centers for Disease Control and Prevention (CDC) and US Department of Agriculture Animal and Plant Health Inspection Service (USDA APHIS), regulates the use and transport of toxic and infectious agents of high consequence, known as biological select agents or toxins (BSAT). ${ }^{1}$ The list of BSAT is maintained based on whether an agent has the ability to severely threaten human life, the lives of domestic plants or animals, or plant or animal products. ${ }^{1}$ Facilities working with these agents must be registered with the FSAP; abide by strict regulations related to safety, security, and data management; and conduct all work with the hazardous agents or products in specially designated areas. ${ }^{2-4}$ It is not unusual, however, for laboratories to move BSAT material, after chemical or heat inactivation, out of FSAP space and into laboratories (typically
BSL-2 class), where requirements for personnel screening, air handling, and decontamination are much less stringent. ${ }^{5}$

In March 2017, FSAP issued a new regulation that requires an in-house validated method of inactivation for any BSAT that is moved out of select agent registered space. ${ }^{6}$ The rule is to ensure that the efficacy of any procedure currently in use to inactivate infectious material can be demonstrated and documented. The inability to take material that formerly contained select agents out of FSAP registered space can have a negative

\footnotetext{
' U.S. Geological Survey National Wildlife Health Center, Madison, WI, USA

Corresponding Author:

Katrina Alger, U.S. Geological Survey National Wildlife Health Center, 6006 Schroeder Road, Madison, WI 537II, USA.

Email: kalger@usgs.gov
} 
impact on workflow efficiency in laboratories limited by space, layout, or equipment placement. As a result, it is critical that laboratories examine the methods they use to inactivate BSAT material to maintain current practices while also complying with the new regulation.

Prior studies have documented the use of heat and/or chemical inactivation of different viruses but have also highlighted potential negative impacts on the efficacy of these treatments caused by variation in virus type or sample matrix. ${ }^{7-10}$ Recently, $\mathrm{Ngo}$ et $\mathrm{al}^{8}$ demonstrated the reliability of MagMAX ${ }^{\mathrm{TM}}$ lysis buffer to inactivate Eastern Equine Encephalitis (EEE), West Nile virus (WNV) and La Crosse virus $(\mathrm{LACV})$ in viral titers as high as $8.1 \log _{10}$ plaque-forming units per milliliter $(\mathrm{PFU} / \mathrm{mL})$, but the authors acknowledged the importance of continued testing to assure these results are consistent for different assay protocols and sample types. For example, their study demonstrated inactivation by inoculating stock virus, suspended in growth media and treated with lysis buffer, into African green monkey kidney (Vero) cells and monitoring the cells for cytopathic effect caused by viral replication. ${ }^{8}$ However, some viruses, such as highly pathogenic avian influenza virus (HPAIV) and virulent Newcastle disease virus (vNDV) are grown in embryonated chicken eggs, ${ }^{11}$ and because characterization of the viral strain typically occurs after virus isolation, archival material for these 2 agents is frequently amnio-allantoic fluid (AAF), not cell culture media. This protein-rich starting material could impede the ability of the lysis buffer to completely inactivate all viable virus. Similarly, heat is known to inactivate viruses, ${ }^{12,13}$ although studies have documented that different viruses have varying thermostability, making it important to verify that heat inactivation procedures are optimized and validated for the specific type of virus and sample matrix being treated. ${ }^{9,10,13}$

Here we demonstrate a validated inactivation protocol for surrogates of HPAIV and vNDV based on the work by Ngo et $\mathrm{al}^{8}$ and adjusted for use of AAF as a culture medium. In addition, we offer clear evidence that the MagMAX ${ }^{\mathrm{TM}}$ extraction kit and protocol, when used correctly, eliminates infectious material from the final nucleic acid elute. Finally, we demonstrate the effectiveness of a procedure to heat inactivate sera taken from animals infected with HPAIV. These protocols can be adopted by any entity needing to validate inactivation for these viruses.

\section{Methods and Materials}

\section{Isolate Selection and Experimental Design}

We completed 3 different experiments to validate inactivation of vNDV and HPAIV surrogates. First, we explored the effectiveness of MagMAX ${ }^{\mathrm{TM}}$ lysis buffer to inactivate vNDV and HPAIV so further extraction steps could be completed in unregistered clean space. Second, we investigated whether MagMAX ${ }^{\mathrm{TM}}-96$ AI/ND Viral RNA Isolation Kit (Thermo Fisher Scientific, Vilnius, Lithuania), when used as labeled, successfully removed all vNDV and HPAIV infectious material from the final nucleic acid eluate. Lastly, we evaluated heat treatments to inactivate HPAIV virus in sera.

Surrogate isolates from the same groups of viruses were chosen from stock archives. Avian paramyxovirus subtype-1 (APMV-1) and H5N2 low pathogenic avian influenza (LPAIV) are nonvirulent strains of the select agents Newcastle disease virus and HPAIV, respectively. ${ }^{14}$ These nonvirulent surrogates are molecularly identical to their virulent counterparts with the exception of short amino acid sequences in each that, when present, result in more severe, systemic infection. ${ }^{14}$ Using surrogates for the select agents of interest allowed laboratory personnel to avoid working directly with select agent material; this is deemed acceptable according to the guidelines for inactivation validation provided by FSAP. ${ }^{2-4}$ Isolates were chosen based on amount of archival material and their high viral load as confirmed through virus titration or quantitative reverse transcriptase PCR (qRT-PCR). In quantitative qRT-PCR, the cycle threshold $(\mathrm{Ct})$ value is inversely related to the starting amount of target DNA, meaning that lower $\mathrm{Ct}$ values correspond to higher amounts of virus in the original sample. ${ }^{15}$ In addition, qRT-PCR product amplification is exponential, meaning very small amounts of target DNA typically result in $\mathrm{Ct}$ values between 35 and 40, while large amounts of target DNA often result in $\mathrm{Ct}$ values of 25 or below. ${ }^{15}$

For the lysis inactivation experiment, the APMV-1 isolate was APMV1/blue-winged teal/Minnesota/204127/2015 in $\mathrm{AAF}$, which had an approximate titer of $1 \times 10^{8.8} \mathrm{EID}_{50} / \mathrm{mL}$, and the H5N2 LPAIV isolate was A/Turkey/Minnesota/3689$1881 / 1981$ in viral transport medium (VTM), which had an approximate titer of $1.33 \times 10^{7} \mathrm{EID}_{50} / \mathrm{mL}$ (Table 1). The MagMAX $^{\mathrm{TM}}$ extraction kit procedure used A/Northern Pintail/California/44242-758/2006 H5N2 LPAIV isolate in AAF with an approximate titer of $1 \times 10^{5} \mathrm{EID}_{50} / \mathrm{mL}$ as well as 15 archival APMV-1 isolates in AAF with $\mathrm{Ct}$ values ranging from 13.45 to 18.12 (Table 1). The qRT-PCR assays we used are highly optimized by the National Animal Health Laboratory Network (NAHLN) - any Ct value below 40 is considered positive, and a Ct below 35 is considered strongly positive (samples with no target DNA present are not expected to amplify at all). ${ }^{16}$ The $\mathrm{Ct}$ values for our isolates are, in comparison, extremely low, indicating very large starting quantities of virus. ${ }^{15}$ For testing heat inactivation of HPAIV in sera, fetal bovine serum (FBS) was spiked with A/northern pintail/ California/44242-758/2006 H5N2 LPAIV to a concentration of $1 \times 10^{5} \mathrm{EID}_{50} / \mathrm{mL}$ (Table 1). The viral loads from all starting material were much higher than what is typically found in routine diagnostic or surveillance samples and therefore believed to provide a robust test for inactivation.

For each trial (except for the nucleic acid extracts), aliquots of the original starting material were reserved to use as the positive controls. For the nucleic acid extracts, the starting material used for inoculation (final eluate) was expected to not contain viable virus, so our in-house positive virus isolation control (H5N2 LPAIV isolate was A/Turkey/Minnesota/ 3689-1881/1981 in VTM) was used instead for the egg inoculation, and a positive amplification control provided by 
Table I. Viral Strains, Matrix, Starting Titer (Measured by 50\% Embryo Infectious Dose [EID 50 ] or qPCR Cycle Threshold Value), and Experimental Usage for all Surrogate Samples Used in Inactivation Validation Testing.

\begin{tabular}{|c|c|c|c|c|}
\hline Agent & Surrogate Viral Strain & Matrix & Starting Titer/Ct Value & Experiment \\
\hline HPAIV & A/turkey/Minnesota/3689-I88I/I98I (H5N2 LPAIV) & VTM & $1.33 \times 10^{7} \mathrm{EID}_{50} / \mathrm{mL}$ & Lysis inactivation \\
\hline HPAIV & A/Northern_Pintail/California/44242-758/2006 & FBS & $\mathrm{I} \times 10^{5} \mathrm{EID}_{50} / \mathrm{mL}$ & Heat inactivation \\
\hline vNDV & APMVI/mallard/Wisconsin/200983/2015 & AAF & $C t=15.73$ & MagMAX'M extraction kit \\
\hline vNDV & APMVI/mallard/Wisconsin/200984/20I5 & AAF & $C t=15.48$ & MagMAX'M extraction kit \\
\hline vNDV & APMVI/mallard/Minnesota/205459/20I5 & AAF & $C t=15.92$ & MagMAX'M extraction kit \\
\hline vNDV & APMVI/blue-winged_teal/Wisconsin/206947/20I5 & AAF & $C t=16.88$ & MagMAX'M extraction kit \\
\hline vNDV & APMVI/blue-winged_teal/Minnesota/204I27/2015 & AAF & $C t=13.45$ & MagMAX'M extraction kit \\
\hline vNDV & APMVI/mallard/Minnesota/203026/20I5 & AAF & $C t=15.85$ & MagMAX'M extraction kit \\
\hline vNDV & APMVI/blue-winged_teal/Louisiana/204552/20I5 & AAF & $C t=16.09$ & MagMAX'M extraction kit \\
\hline vNDV & APMVI/blue-winged_teal/Louisiana/204348/20I5 & AAF & $C t=16.42$ & MagMAX'M extraction kit \\
\hline vNDV & APMVI/american_green-winged_teal/Delaware/22048I/20I6 & AAF & $C t=16.43$ & MagMAX'M extraction kit \\
\hline
\end{tabular}

AAF, amnio-allantoic fluid; APMVI, avian paramyxovirus subtype-I; Ct, cycle threshold; FBS, fetal bovine serum; HPAIV, highly pathogenic avian influenza; vNDV, virulent Newcastle disease virus; VTM, viral transport media.

NAHLN was used for the qRT-PCR. Throughout all trials, negative controls consisting of sterile VTM were included. Due to the limited availability of eggs, negative controls used fewer eggs than positive controls in some trials. Given the nature of the experiments and the fact that a false negative would be considered more consequential than false positives, we felt that shorting the negative contamination control was justified in order to have enough eggs to provide confidence that the positive controls were performing as expected.

\section{Lysis Buffer Inactivation Trials}

Nine $50 \mu \mathrm{L}$ aliquots of APMV-1 isolate were made and $101 \mu \mathrm{L}$ of MagMAX ${ }^{\mathrm{TM}}$ lysis buffer added to each, according to the manufacturer guidelines ${ }^{17}$ and the NAHLN standard operating

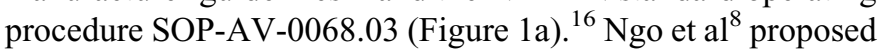
a 10-minute incubation period once the lysis buffer and sample are mixed, prior to inoculation in cell culture. MagMAX ${ }^{\mathrm{TM}}$ lysis buffer contains a protein denaturant, ${ }^{18}$ but given that our starting APMV-1 isolate material of AAF is more protein-rich than cell culture media, we anticipated that 10 minutes might not be sufficient for this sample type. To address this, we tested 3 different lengths of incubation time $(10,20$, and 30 minutes, Figure 1a). The buffer's chemical properties led us to suspect it may be toxic when inoculated directly into embryonated eggs; therefore, following the appropriate incubation times, each aliquot was diluted with VTM to a total volume of $1 \mathrm{~mL}$ (Figure 1a). The solution was mixed by vortexing, after which the entire mixture was inoculated into 8-day-old embryonated chicken eggs (200 $\mu \mathrm{L} /$ egg, 5 egg replicates, Figure 2$)$. Every time-set included a positive control consisting of $50 \mu \mathrm{L}$ of the untreated starting material, diluted to $1 \mathrm{~mL}$ with VTM, and 1 set (3 eggs) of negative controls (sterile VTM) was included for the entire experiment. The initial trial results showed a high degree of variability across all time-sets, so 1 minute of agitation on an orbital shaker was added prior to incubation at room temperature for 30 minutes to ensure complete homogenization of isolate material and lysis buffer (Figure 1b). This extra step of mixing yielded more consistent results; therefore, this revised protocol was used for our subsequent lysis buffer trials.

\section{Virus Isolation and Subsequent Testing}

All trials using embryonated eggs were conducted in a manner consistent with standard methods for virus isolation of avian pathogens. ${ }^{19}$ Eggs were incubated at $37.5^{\circ} \mathrm{C}$ with $50 \%$ relative humidity and turned once an hour for 8 days prior to inoculation. Viable eggs were disinfected using an alcohol/iodine mixture, and a small hole was poked in the shell using a sterilized instrument. The hole was resterilized with alcohol/iodine, and then the egg was inoculated with $200 \mu \mathrm{L}$ of sample material using a 27 -gauge, $1 \mathrm{~mL}$ tuberculin syringe, after which the hole was sealed with a small drop of glue to prevent evaporation. Inoculated eggs were incubated, without turning, at $37.5^{\circ} \mathrm{C}$ with $50 \%$ relative humidity for 3 days while egg death was monitored and recorded. At the end of 3 days, the eggs were chilled at $4^{\circ} \mathrm{C}$ for 4 hours prior to recovery of the AAF.

Testing the AAF for hemagglutinating agents (HA) was done at the time of collection, and real-time qRT-PCR for the AIV and APMV-1 matrix genes took place after RNA extraction. Each egg was harvested and tested individually. To test for HA, $6 \mathrm{~mL}$ of rooster red blood cells (RBCs) in Alsever's 


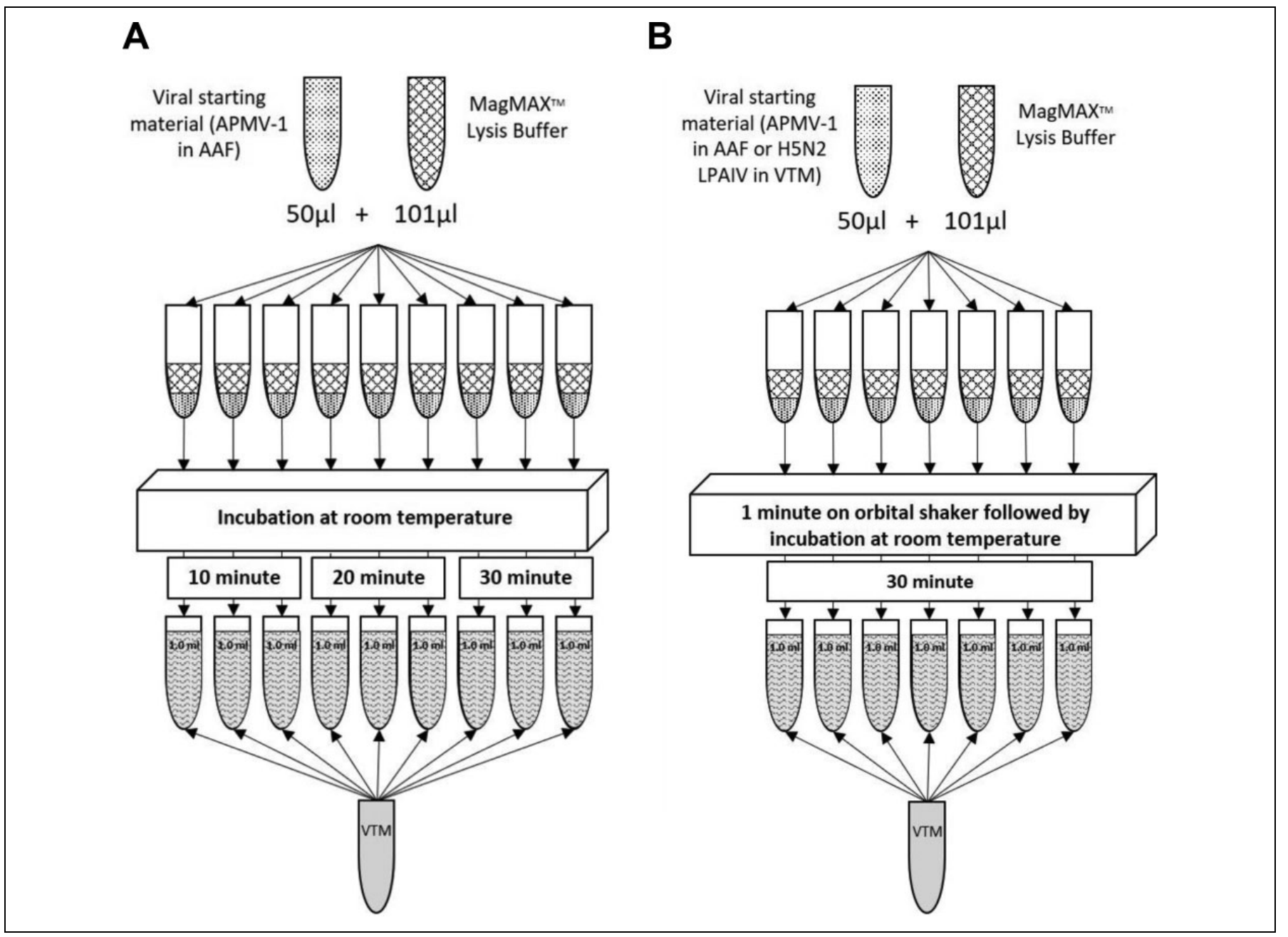

Figure I. Illustrated workflow for MagMAXTM lysis buffer inactivation of APMV-I and H5N2 LPAIV in AAF. (a) Workflow for initial trial (APMV-I only) using variable incubation times at room temperature, prior to dilution with VTM and inoculation into 8-day old embryonated chicken eggs. (b) Workflow for all subsequent trials (APMV-I and H5N2 LPAIV) adding I minute of agitation on an orbital shaker, followed by 30 minutes of incubation at room temperature, prior to dilution with VTM and inoculation into 8-day old embryonated chicken eggs. AAF, amnio-allantoic fluid; APMV-I, avian paramyxovirus subtype-I; LPAIV, low pathogenic avian influenza; VTM, viral transport media.

solution was gently mixed with approximately $8 \mathrm{~mL}$ of phosphate-buffered saline (PBS) in a sterile $15 \mathrm{~mL}$ conical tube. The cells were washed 3 times by centrifugation $(1500$ $\mathrm{rpm}$ or $423 \mathrm{rcf}$ for 10 minutes at $4^{\circ} \mathrm{C}$ ) followed, each time, by the removal and replacement of the PBS supernatant. The remaining packed $\mathrm{RBC}$ volume after washing was approximately $1 \mathrm{~mL} ; 9 \mathrm{~mL}$ of PBS was then added to create a $10 \%$ solution. This solution was then further diluted to a $0.5 \%$ solution, and using a U-bottom microtiter plate, $50 \mu \mathrm{L}$ was combined with $50 \mu \mathrm{L}$ of AAF from each egg. The plate was incubated at $4^{\circ} \mathrm{C}$ for 30 minutes, after which it was read for evidence of hemagglutinating agents, indicated by RBC remaining in suspension rather than forming a bead at the bottom of the plate well ${ }^{20}$, and results recorded. Nucleic acid extraction and qRT-PCR was performed in accordance with NAHLN protocol SOP-AV-0068.03. ${ }^{16}$

Optimal incubation time for inactivation was determined by residual viral viability, assessed based on embryo death, HA results, and final qRT-PCR Ct value. Once optimal incubation time was determined, the process was repeated, at that incubation time, to confirm the results of the previous trial. For the final trials testing inactivation of both APMV-1 and LPAIV, a sample size of 35 was calculated as the number of eggs needed for $95 \%$ confidence that the inactivation was $>90 \%$ effective, given an expected prevalence of zero (http://sampsize.source forge.net, Version 0.6, 2003). Samples were prepared as previously described using seven $50-\mu \mathrm{L}$ aliquots of starting material to which $101 \mu \mathrm{L}$ of lysis buffer was added. Following 1 minute of agitation on an orbital shaker and 30 minutes of incubation at room temperature, each of the 7 samples was diluted to $1 \mathrm{~mL}$ using VTM and inoculated into 5 eggs $(200 \mu \mathrm{L}$ per egg; Figures $1 \mathrm{~b}$ and 2). Also included were a positive control consisting of $50 \mu \mathrm{L}$ of the untreated starting material, diluted to 1 $\mathrm{mL}$ with VTM, and a negative control of sterile VTM-each inoculated into 5 eggs (Figure 2). Material was passaged twice (1 egg to 1 egg), in accordance with in-house protocol for the 


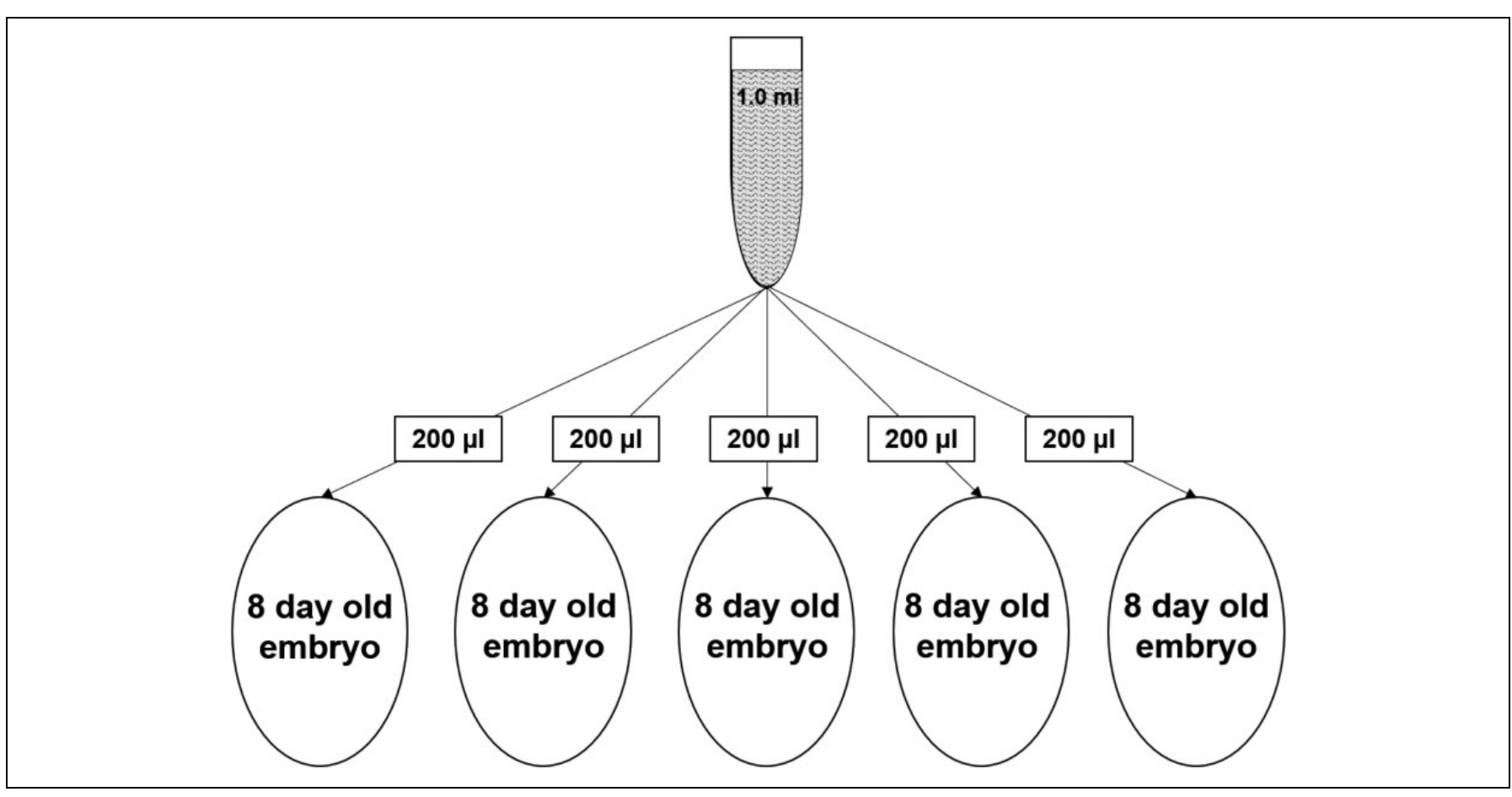

Figure 2. Illustrated workflow for egg inoculation in MagMAX ${ }^{\mathrm{TM}}$ lysis buffer inactivation trials (avian paramyxovirus subtype-I and H5N2 low pathogenic avian influenza).

isolation of avian influenza viruses and avian paramyxoviruses. Embryo deaths, HA results, and final $\mathrm{Ct}$ values were noted for each round of isolation.

\section{Inactivation of Virus in Nucleic Acid Extracts}

Because isolating and characterizing a virus may occur after initial extraction, we conducted an experiment to verify that the extraction procedure for unknown viruses also removes any viable virus using conventionally extracted nucleic acid as the starting material. To investigate inactivation of APMV-1 by routine extraction, 15 known viable APMV-1 isolates were removed from archive and extracted, using the MagMAX ${ }^{\mathrm{TM}}$ $96 \mathrm{AI} / \mathrm{ND}$ Viral RNA Isolation Kit, in $50-\mu \mathrm{L}$ aliquots, following the NAHLN protocol SOP-AV-0068.03. ${ }^{16}$ Isolates were extracted in duplicate for a total of 30 samples. An aliquot of $8 \mu \mathrm{L}$ from each extract was tested for the APMV-1 matrix gene by qRT-PCR, and the initial $\mathrm{Ct}$ values, ranging from 13.39 to 18.13, were recorded (Table 1 ). The remaining $42 \mu \mathrm{L}$ were added to $200 \mu \mathrm{L}$ of VTM to create sufficient volume for egg inoculation, and the entire amount was then inoculated into a single egg (for a total of 30 eggs). Egg inoculation, monitoring, and testing followed the same procedures outlined previously, with the exception that HA testing was performed in triplicate for each sample. Samples were passaged twice (1 egg to $1 \mathrm{egg}$ ).

To investigate inactivation of H5N2 LPAIV by routine extraction, stock virus was diluted in VTM to $1 \times 10^{5} \mathrm{EID}_{50} /$ $\mathrm{mL}$, then extracted into ten $50-\mu \mathrm{L}$ aliquots following the MagMAX ${ }^{\mathrm{TM}}$ Viral RNA Isolation Kit manufacturer recommendations. ${ }^{17}$ Each sample was diluted 1:4 in PBS to obtain sufficient volume for egg inoculation $(200 \mu \mathrm{L})$ and then inoculated into a single egg. Egg inoculation, monitoring, and HA testing followed the same procedures outlined previously. Samples were passaged once (1 egg to 1 egg). Positive and negative controls for each nucleic acid extract trial were included as described earlier.

\section{Heat Inactivation of LPAIV in FBS}

Stock LPAIV was diluted in FBS to $10^{5} \mathrm{EID}_{50} / \mathrm{mL}$, and this solution was then aliquoted into $1-\mathrm{mL}$ fractions. Heat inactivation testing was conducted for 3 different conditions: 30 minutes in a $56^{\circ} \mathrm{C}$ water bath, 60 minutes in a $56^{\circ} \mathrm{C}$ water bath, and 30 minutes in a $70^{\circ} \mathrm{C}$ water bath. Treatment conditions were applied to 10 aliquots, with a single aliquot left untreated as a control. Each aliquot was inoculated into 5 eggs ( $200 \mu \mathrm{L} / \mathrm{egg})$. Inoculated eggs were incubated, without turning, at $37.5^{\circ} \mathrm{C}$ with $50 \%$ relative humidity for 3 days, and egg death was monitored and recorded. At the end of 3 days, the eggs were all chilled at $4^{\circ} \mathrm{C}$ for 4 hours prior to harvesting the AAF. Harvested AAF were HA tested following the protocol outlined previously.

Any treatment that yielded HA positive AAF received no further testing; treatments that only produced HA negative AAF were passed into a second round of embryonated eggs (1 egg to 1 egg). In addition, 5 eggs were inoculated with 200 $\mu \mathrm{L}$ of FBS as a negative control, and 5 eggs were inoculated with $200 \mu \mathrm{L}$ of FBS containing $10^{5} \mathrm{EID}_{50} / \mathrm{mL}$ LPAI virus as a positive control. Eggs were incubated, harvested, and HA tested as previously described. After HA testing, all samples 
Table 2. Effect of Variable Incubation Times at Room Temperature on Inactivation of Avian Paramyxovirus Subtype-I (APMV-I) in Chicken AmnioAllantoic Fluid (AAF) by MagMAX'M Lysis Buffer, as Measured by Hemagglutination Activity (HA) and Cycle Threshold (Ct) qRT-PCR Results. ${ }^{\text {a }}$

\begin{tabular}{|c|c|c|c|c|c|c|c|c|}
\hline \multicolumn{3}{|c|}{ I0-Minute Incubation Time } & \multicolumn{3}{|c|}{ 20-Minute Incubation Time } & \multicolumn{3}{|c|}{ 30-Minute Incubation Time } \\
\hline I & - & No $C_{t}$ & I & - & 35.93 & 1 & - & No $\mathrm{Ct}$ \\
\hline 2 & - & No $C t$ & 2 & - & 35.6 & 2 & - & 35.58 \\
\hline 3 & - & 35.77 & 3 & - & 34.89 & 3 & - & No Ct \\
\hline 4 & - & No $C t$ & 4 & - & 34.84 & 4 & - & 37.89 \\
\hline 7 & - & 22.15 & 7 & - & 37.24 & 7 & - & No $C t$ \\
\hline 8 & - & 24.89 & 8 & - & 35.38 & 8 & - & No $C t$ \\
\hline 9 & - & 24.11 & 9 & - & No Ct & 9 & - & No Ct \\
\hline 10 & - & 23.32 & 10 & - & 36.9 & 10 & - & $\mathrm{No} C t$ \\
\hline 11 & - & No $C_{t}$ & 11 & - & No Ct & II & - & No $C t$ \\
\hline 12 & - & No Ct & 12 & - & No Ct & 12 & + & 13.2 \\
\hline
\end{tabular}

${ }^{a}$ Number of eggs used for controls in parentheses. One set of 3 eggs inoculated with viral transport media were included as negative controls across all time-series.

${ }^{b}$ Result of positive control incubating for 10 minutes established in earlier trial (data not shown) and therefore not repeated to allow adequate positive controls for longer incubation times.

were further tested for the presence of LPAIV nucleic acid by qRT-PCR.

\section{Results}

\section{Lysis Buffer APMV-I Inactivation}

Initial trials to pinpoint optimal incubation time for APMV-1 inactivation in lysis buffer yielded inconsistent results. Although embryo death across all 3 incubation times was minimal, HA positive eggs were present in all 3 sample sets (Table 2). In the 10-minute incubation time group, 1 of 15 embryos (approximately 7\%) tested HA positive. In the 20minute incubation time group, 1 of 15 eggs (approximately $7 \%$ ) died on day 3 and tested HA positive. In the 30-minute incubation group, 4 of 15 embryos (approximately 27\%) tested HA positive, and 1 of those 4 embryos died on day 2. Subsequent qRT-PCR testing also showed inconsistency within all 3 time-sets, indicated by a high number of samples having $\mathrm{Ct}$ values below 25 (a strong amplification signal unlikely to be the result of residual nucleic acid alone): approximately $33 \%$ $(5 / 15)$ of eggs in the 10-minute group, $7 \%(1 / 15)$ of eggs in the 20 -minute group, and $27 \%(4 / 15)$ of eggs in the 30-minute group (Table 2). Controls performed as expected, eliminating concerns of contamination.

Repeating the trial with the addition of agitation for 1 minute on an orbital shaker prior to 30-minute incubation yielded results that were more consistent and aligned better with expected results for successful viral inactivation. Of 15 eggs tested, 1 embryo died on the first day, and there were no HA positive results (Table 3). Embryos that died within 24 hours of inoculation were not considered reliable indicators of viral growth due to the higher likelihood that death was caused by trauma during inoculation, stress on the embryo, or bacterial contamination. ${ }^{21}$ Subsequent qRT-PCR testing showed Ct values for each sample that ranged from 30.23 to 37.24 (Table 3). One round of passage resulted in no embryo deaths, no HA positive results, and qRT-PCR Ct values in approximately $67 \%$ $(10 / 15)$ of samples, ranging from 36.38 to 39.28 , with approximately $34 \%(5 / 10)$ of samples having no amplification at all (Table 3). A second, final passage resulted in no embryo death, no HA positive results, and no amplification for any sample by qRT-PCR (Table 3).

Testing 20 additional eggs (to increase sample size) using the same protocol yielded similar results as the previous trial. Initial inoculation resulted in 2 embryo deaths ( 1 on day 1 and the other on day 3), no HA positive results, $\mathrm{Ct}$ values for $20 \%(3 / 15)$ of samples above 39 , and $80 \%(12 /$ 15) of samples showing no amplification at all (Table 4). One round of passage resulted in no embryo death, no HA positive results, and no amplification for any sample by qRT-PCR (Table 4). Due to the lack of evidence for the presence of any viable virus in any sample, a second passage was not completed.

\section{Lysis Buffer LPAIV Inactivation}

Results for the test of LPAIV inactivation in lysis buffer were consistent with the APMV-1 trials. Initial inoculation resulted in 2 embryo deaths ( 1 on day 2 and the other on day 3 ), no HA positive results, $\mathrm{Ct}$ values for approximately $5.7 \%(2 / 35)$ of 
Table 3. Effect of Adding I Minute of Agitation on an Orbital Shaker Prior to 30-Minute Incubation at Room Temperature on Inactivation of Avian Paramyxovirus Subtype-I in Chicken Amnio-Allantoic Fluid by MagMAX'M Lysis Buffer, as Measured by Hemagglutination Activity (HA), and Cycle Threshold (Ct) qPCR Results: Trial I, Comprised of I5 Replicates.

\begin{tabular}{|c|c|c|c|c|c|c|c|c|}
\hline \multicolumn{3}{|l|}{ Original Inoculation } & \multicolumn{3}{|c|}{ First Passage } & \multicolumn{3}{|c|}{ Second Passage } \\
\hline Egg No. & HA & qPCR Ct & Egg No. & $\mathrm{HA}$ & qPCR Ct & Egg No. & $\mathrm{HA}$ & qPCR Ct \\
\hline I & - & 30.7 & I & - & 39.28 & I & - & No Ct \\
\hline 2 & - & 32.36 & 2 & - & No Ct & 2 & - & $\mathrm{No} C t$ \\
\hline 3 & - & 31.73 & 3 & - & 38.2 & 3 & - & No Ct \\
\hline 4 & - & 30.52 & 4 & - & 37.12 & 4 & - & No Ct \\
\hline 5 & - & 32.68 & 5 & - & 36.38 & 5 & - & $\mathrm{No} C t$ \\
\hline 6 & - & 30.6 & 6 & - & 37.21 & 6 & - & No Ct \\
\hline 7 & - & 32.09 & 7 & - & 38.4 & 7 & - & $\mathrm{No} C t$ \\
\hline 8 & - & 30.23 & 8 & - & 38.17 & 8 & - & No Ct \\
\hline 9 & - & 31.58 & 9 & - & 37.48 & 9 & - & $\mathrm{No} C t$ \\
\hline 10 & - & 32.14 & 10 & - & $\mathrm{No} C t$ & 10 & - & $\mathrm{No} C t$ \\
\hline II & - & 30.93 & II & - & 38.27 & II & - & $\mathrm{No} C t$ \\
\hline 12 & - & 30.72 & 12 & - & No Ct & 12 & - & $\mathrm{No} C t$ \\
\hline 13 & - & 36.49 & 13 & - & No Ct & 13 & - & $\mathrm{No} C t$ \\
\hline 14 & - & 32.81 & 14 & - & $\mathrm{No} C t$ & 14 & - & $\mathrm{No} C t$ \\
\hline 15 & - & 37.24 & 15 & - & 38.54 & 15 & - & $\mathrm{No} \mathrm{Ct}_{t}$ \\
\hline Positive control (5) & All + & Average 14.56 & Positive control (5) & All + & Average 13.12 & Positive control (5) & All + & Average 14.46 \\
\hline Negative control (3) & All - & $\mathrm{No} C t$ & Negative control (3) & All - & $\mathrm{No} C t$ & Negative control (3) & All - & No $\mathrm{Ct}$ \\
\hline
\end{tabular}

${ }^{\mathrm{a}}$ Number of eggs used for controls in parentheses.

Table 4. Effect of Adding I Minute of Agitation on an Orbital Shaker Prior to 30-Minute Incubation at Room Temperature on Inactivation of Avian Paramyxovirus Subtype-I in Chicken Amnio-Allantoic Fluid by MagMAX ${ }^{\mathrm{TM}}$ Lysis Buffer, as Measured by Hemagglutination Activity (HA) and Cycle Threshold (Ct) qPCR Results: Trial 2, Comprised of 20 Additional Replicates (identical procedure). ${ }^{\text {a }}$

\begin{tabular}{|c|c|c|c|c|c|}
\hline \multicolumn{3}{|l|}{ Original Inoculation } & \multicolumn{3}{|c|}{ First Passage } \\
\hline Egg No. & $\mathrm{HA}$ & $\mathrm{qPCR} C \mathrm{t}$ & Egg No. & $\mathrm{HA}$ & qPCR Ct \\
\hline I & - & $\mathrm{No} C t$ & I & - & No Ct \\
\hline 2 & - & $\mathrm{No} C t$ & 2 & - & No Ct \\
\hline 3 & - & No Ct & 3 & - & $\mathrm{No} C t$ \\
\hline 4 & - & No Ct & 4 & - & No Ct \\
\hline 5 & - & 39.34 & 5 & - & No Ct \\
\hline 6 & - & $\mathrm{No} C t$ & 6 & - & No Ct \\
\hline 7 & - & No Ct & 7 & - & No Ct \\
\hline 8 & - & No Ct & 8 & - & No Ct \\
\hline 9 & - & 39.4 & 9 & - & No Ct \\
\hline 10 & - & $\mathrm{No} C t$ & 10 & - & No Ct \\
\hline II & - & No Ct & II & - & No Ct \\
\hline 12 & - & No Ct & 12 & - & $\mathrm{No} C t$ \\
\hline 13 & - & $\mathrm{No} C t$ & 13 & - & No Ct \\
\hline 14 & - & No Ct & 14 & - & $\mathrm{No} C t$ \\
\hline 15 & - & No Ct & 15 & - & No Ct \\
\hline 16 & - & No Ct & 16 & - & No Ct \\
\hline 17 & - & No Ct & 17 & - & $\mathrm{No} C t$ \\
\hline 18 & - & $\mathrm{No} C t$ & 18 & - & No Ct \\
\hline 19 & - & No Ct & 19 & - & No Ct \\
\hline 20 & - & 39.78 & 20 & - & $\mathrm{No} C \mathrm{t}$ \\
\hline Positive control (5) & All + & Average 16.02 & Positive control (3) & All + & Average 12.33 \\
\hline Negative control (3) & All - & $\mathrm{No} C t$ & Negative control (3) & All - & No Ct \\
\hline
\end{tabular}

${ }^{a}$ Number of eggs used for controls in parentheses.

samples over 40, and approximately $94 \%$ (33/35) showing no amplification at all (Table 5). After 1 passage, there was no embryo death, no HA positive results, and no amplification in any sample (Table 5). Due to the lack of evidence for the presence of any viable virus in any sample, a second passage was not completed. 
Table 5. Effect of 30-Minute Incubation at Room Temperature, Preceded by I Minute of Agitation on an Orbital Shaker, on Inactivation of H5N2 Low Pathogenic Avian Influenza in Chicken Amnio-Allantoic Fluid by MagMAX ${ }^{\text {TM }}$ Lysis Buffer, as Measured by Hemagglutination Activity (HA) and Cycle Threshold (Ct) qPCR Results. ${ }^{a}$

\begin{tabular}{|c|c|c|c|c|c|}
\hline \multicolumn{3}{|c|}{ ORIGINAL INOCULATION } & \multicolumn{3}{|c|}{ FIRST PASSAGE } \\
\hline Egg No. & $\mathrm{HA}$ & qPCR Ct & Egg No. & $\mathrm{HA}$ & qPCR Ct \\
\hline 1 & - & No Ct & 1 & - & No Ct \\
\hline 2 & - & $\mathrm{No} C t$ & 2 & - & No Ct \\
\hline 3 & - & No Ct & 3 & - & No Ct \\
\hline 4 & - & $\mathrm{No} C t$ & 4 & - & No Ct \\
\hline 5 & - & No Ct & 5 & - & No Ct \\
\hline 6 & - & $\mathrm{No} C t$ & 6 & - & No Ct \\
\hline 7 & - & No Ct & 7 & - & No Ct \\
\hline 8 & - & $\mathrm{No} C t$ & 8 & - & No Ct \\
\hline 9 & - & No Ct & 9 & - & No Ct \\
\hline 10 & - & $\mathrm{No} C t$ & 10 & - & No Ct \\
\hline II & - & $\mathrm{No} C t$ & 11 & - & No Ct \\
\hline 12 & - & $\mathrm{No} C t$ & 12 & - & No Ct \\
\hline 13 & - & $\mathrm{No} C t$ & 13 & - & No Ct \\
\hline 14 & - & No Ct & 14 & - & $\mathrm{No} C t$ \\
\hline 15 & - & $\mathrm{No} C t$ & 15 & - & No Ct \\
\hline 16 & - & No Ct & 16 & - & $\mathrm{No} C t$ \\
\hline 17 & - & $\mathrm{No} C t$ & 17 & - & No Ct \\
\hline 18 & - & No Ct & 18 & - & $\mathrm{No} C t$ \\
\hline 19 & - & $\mathrm{No} C t$ & 19 & - & No Ct \\
\hline 20 & - & $\mathrm{No} C t$ & 20 & - & No Ct \\
\hline 21 & - & 40.44 & 21 & - & $\mathrm{No} C t$ \\
\hline 22 & - & $\mathrm{No} C t$ & 22 & - & No Ct \\
\hline 23 & - & 40.39 & 23 & - & No Ct \\
\hline 24 & - & $\mathrm{No} C t$ & 24 & - & No Ct \\
\hline 25 & - & No Ct & 25 & - & $\mathrm{No} C t$ \\
\hline 26 & - & $\mathrm{No} C t$ & 26 & - & $\mathrm{No} C t$ \\
\hline 27 & - & $\mathrm{No} C t$ & 27 & - & No Ct \\
\hline 28 & - & No Ct & 28 & - & $\mathrm{No} C t$ \\
\hline 29 & - & $\mathrm{No} C t$ & 29 & - & No Ct \\
\hline 30 & - & No Ct & 30 & - & $\mathrm{No} C t$ \\
\hline 31 & - & $\mathrm{No} C t$ & 31 & - & $\mathrm{No} C t$ \\
\hline 32 & - & No Ct & 32 & - & $\mathrm{No} C t$ \\
\hline 33 & - & $\mathrm{No} C t$ & 33 & - & No Ct \\
\hline 34 & - & $\mathrm{No} C t$ & 34 & - & $\mathrm{No} C t$ \\
\hline 35 & - & No Ct & 35 & - & No Ct \\
\hline Positive control (3) & All + & Avg. 11.93 & Positive control (3) & All + & Average 11.97 \\
\hline Negative control (3) & All - & No $\mathrm{Ct}$ & Negative control (2) & All - & No Ct \\
\hline
\end{tabular}

${ }^{a}$ Number of eggs used for controls in parentheses.

\section{APMV-I and LPAIV Inactivation in Nucleic Acid Extracts}

The AAF harvested from all eggs inoculated with APMV-1 nucleic acid extract tested HA negative in triplicate. No embryos died during the course of incubation, and subsequent qRT-PCR revealed no amplification from any sample. After 1 passage, all eggs continued to test HA-negative in triplicate. Similarly, there were no embryo deaths during the course of incubation, and subsequent qRT-PCR revealed no amplification from any sample, although positive and negative controls performed as expected. The AAF harvested from all eggs inoculated with LPAIV nucleic acid extract tested HA negative. After 1 passage, all samples remained HA negative. No further testing was conducted.

\section{Heat Inactivation of LPAIV in FBS}

The AAF of all eggs inoculated with serum aliquots that underwent heat inactivation in the first treatment group (30 minutes at $56^{\circ} \mathrm{C}$ ) tested positive for the presence of hemagglutinating agents. Similarly, the AAF of eggs inoculated with serum aliquots from the second heat inactivation treatment group (60 minutes at $56^{\circ} \mathrm{C}$ ) were almost entirely positive for the presence of hemagglutinating agents, with only 1 egg out of 50 testing HA negative. All AAF from eggs inoculated with serum aliquots in the third treatment group $\left(30\right.$ minutes at $\left.70^{\circ} \mathrm{C}\right)$ tested negative for hemagglutinating agents. After a single passage, all AAF remained HA negative, and subsequent testing by qRT-PCR was also negative for LPAIV. 


\section{Discussion}

All diagnostic and research laboratories physically compartmentalize different aspects of their work with infectious material. Reasons include the need to limit personnel access to certain spaces for purposes of biosafety or security, good laboratory practice such as maintaining unidirectional workflow, and limitations of facility space, laboratory layout, or equipment placement. Adherence to appropriate biosafety practices is critical for laboratories working with any known pathogens or high-risk material. When working with samples containing BSAT, extra precautions must be taken to ensure the safety of laboratory personnel and minimize potential risk to agriculture or public health. ${ }^{1}$ Recent regulations passed by FSAP are meant to provide an additional layer of security by requiring laboratories to follow a validated inactivation procedure for any known BSAT-containing sample material that is moved out of an FSAP registered space for storage or continued work. ${ }^{2-4}$ In recognition that many laboratories perform different sample-processing steps or molecular characterization in designated areas that may or may not be registered FSAP space, we sought to demonstrate the effectiveness of several inactivation methods and establish validated procedures that meet the FSAP guidance, provide reasonable assurance that no infectious BSAT material leaves FSAP-registered space, and minimize workflow disruption.

Our trials demonstrate that MagMAX ${ }^{\mathrm{TM}}$ lysis buffer effectively inactivates APMV-1 and LPAI viruses (surrogates for vNDV and HPAIV) in AAF with some small adjustments to the manufacturer's protocol. This protocol, when applied to known select agent infected material, has been updated to include 30 minutes of sample incubation in lysis buffer preceded by 1 minute of agitation on an orbital shaker. The original manufacturer protocol also includes a step of agitation after combining the lysis buffer and sample. ${ }^{17}$ It is possible this step is necessary for successful protein denaturation, which would explain why adding it to our protocol prior to incubation yielded greater consistency in our final inactivation results. Although difficult to interpret, some of the results from the initial lysis buffer trials seemed to indicate successful inactivation could also take place at a lower incubation time. We chose to keep the incubation time at 30 minutes in our final standard operating protocol because this length of time is easy to accomplish without compromising the workflow for the rest of the procedure while providing a robust safety margin. Given the types of samples handled and work conducted in our laboratories, this safety margin is believed to outweigh the risk associated with not performing verification viability testing regularly on all samples. Going forward, we plan to review these protocols annually, during the required review by the center's responsible official. Revalidation will occur periodically, after a given number of samples (yet to be determined) have undergone inactivation.

One consistent finding we noted during the lysis buffer trials was the tendency of AAF harvested from eggs on the first and second passage to contain amplifiable viral nucleic acid.
Molecular assays have gained popularity in virology diagnostics because of their speed and sensitivity, but it is important to recognize the limitations of these tests and interpret the results with caution. Amplification of nucleic acid during qRT-PCR will occur whenever there is genetic material in the sample that matches the assay's primer and probe sequences. The presence of genetic material, however, does not necessarily equate to the presence of viable virus. In our study, the fact that APMV-1 and LPAIV RNA was detected in the AAF of eggs after 1 or 2 passages is, almost certainly, the result of residual RNA from the inoculated material that has not yet been broken down by the nuclease enzymes of the egg. This interpretation is substantiated by the fact that there was no other indication of viral infection in the eggs (e.g., high egg death or positive hemagglutination tests) and with each passage, the amount of amplified material steadily decreased and disappeared as the nucleic acid from the original inoculant was broken down and failed to regenerate.

This study also demonstrated the ability of the MagMAX $^{\mathrm{TM}}$ RNA extraction kit and protocol to remove viable virus from the original sample and prevent the presence of any infectious material in the final nucleic acid eluate. Ngo et $\mathrm{al}^{8}$ recently demonstrated the effectiveness of this same kit to inactivate several different viruses, including the select agent EEE virus. Their study differed from ours in both agent and sample matrix tested. While our findings were not necessarily surprising given the earlier study's success, confirming the inactivation effectiveness of the MagMAX ${ }^{\mathrm{TM}}$ kits for the specific materials handled in our laboratories was reassuring and will allow us to continue taking advantage of both off-site sequencing services and on-site storage options in non-FSAP registered spaces.

While HPAIV is not typically a blood-borne pathogen, laboratories using serum from HPAIV infected animals outside of select agent registered space must still demonstrate viral inactivation for that media to comply with new FSAP regulations. In 1990, King ${ }^{10}$ showed that HPAIV, diluted in chicken serum to a starting HA titer as high as $10^{6.8} / \mathrm{mL}$, lost infectivity (determined by culture in embryonated eggs) when heat-treated at $56^{\circ} \mathrm{C}$ for 60 minutes. Based on these results, it is unsurprising that our initial treatment conditions of $56^{\circ} \mathrm{C}$ for 30 minutes were ineffective for inactivating LPAIV in FBS. However, we also found evidence of continued infectivity at 60 minutes, which contradicts the previous study. Further work is needed to investigate the reason for this disparity, but given our unequivocal results at the higher temperature, we are confident that for our purposes, a $70^{\circ} \mathrm{C}$ incubation for 30 minutes will render any HPAIV samples noninfectious. This confidence increases when considering that our test samples and the samples from King ${ }^{10}$ had extremely high starting titers that were achieved by culture and would be unusual to find in biological samples.

Whether culturing diagnostic samples for characterization or conducting research that will help us better understand and manage these pathogens, laboratory protocols should maximize the efficiency of their work while also ensuring the utmost 
adherence to biosafety practices and regulations. To optimize workflow in limited laboratory space while complying with biosafety and security regulations and maintaining unidirectional workflow, it may be necessary to move sample material containing BSAT into nonregistered space. Following the steps outlined in this study will allow those workflows to remain unchanged while also offering reasonable assurance that any sample leaving a registered space no longer contains viable, infectious virus.

\section{Acknowledgments}

The authors are grateful to Dr Tonie Rocke for providing valuable feedback on an earlier draft.

\section{Ethical Approval Statement}

Not applicable to this research.

\section{Statement of Human and Animal Rights}

There were no human subjects involved in this study. All animal work described in this study complies with the National Institutes of Health Office of Laboratory Welfare Public Health Service Policy on Humane Care and Use of Laboratory Animals ("PHS Policy"), which regulates the offspring of egg-laying vertebrate species only after hatching. All chicken embryos used in this study were terminated by day 12 , which is well below the expected hatch age of 21 days. Therefore, according to PHS Policy, approval by the National Wildlife Health Center's Institutional Animal Care and Use Committee was not required.

\section{Statement of Informed Consent}

Not applicable to this research.

\section{Declaration of Conflicting Interests}

The authors declared the following potential conflicts of interest with respect to the research, authorship, and/or publication of this article: The use of trade, product, or firm names is for descriptive purposes only and does not imply endorsement by the U.S. government.

\section{Funding}

The authors disclosed receipt of the following financial support for the research, authorship, and/or publication of this article: This work was funded by the United States Geological Survey, and the National Institute of Allergy and Infectious Diseases, the National Institutes of Health, under contract number HHSN272201400006C.

\section{ORCID iD}

Katrina Alger (D) https://orcid.org/0000-0001-7708-0203

\section{References}

1. Centers for Disease Control and United States Department of Agriculture. Federal Select Agent Program. 2017. https://www. selectagents.gov. Accessed September 16, 2017.

2. Centers for Disease Control and United States Department of Agriculture. Agriculture: possession, use, and transfer of select agents and toxins. Fed Regist. 2005;70 FR 13278. Codified at 7 CFR $\S 331$.
3. Centers for Disease Control and United States Department of Agriculture. Animal and animal products: possession, use, and transfer of select agents and toxins. Fed Regist. 2005;70 FR 13284. Codified at 9 CFR $\S 121$.

4. Centers for Disease Control and United States Department of Agriculture. Public health: possession, use, and transfer of select agents and toxins. Fed Regist. 2005;70 FR 13316. Codified at 42 CFR $\S 73$.

5. United States Department of Health and Human Services, Public Health Service. Biosafety in microbiological and biomedical laboratories. 5th ed. 2009. HHS Pub No. (CDC 21-1112). https://www.cdc.gov/biosafety/publications/bmbl5/BMBL.pdf. Accessed September 17, 2017.

6. Centers for Disease Control and Prevention Division of Select Agents and Toxins and Animal and Plant Health Inspection Services Agriculture Select Agent Services. Guidance on the inactivation or removal of select agents and toxins for future use, 7 CFR Part 331, 9 CFR Part 121.3, 42 CFR Part 73.3. 2017. https://www. selectagents.gov/resources/Inactivation_Guidance.pdf. Accessed September 16, 2017.

7. Blow J, Dohm D, Negley D, Mores C. Virus inactivation by nucleic acid extraction reagents. J Virol Methods. 2004;119(2): 195-198.

8. Ngo K, Jones S, Church $\mathrm{T}$, et al. Unreliable inactivation of viruses by commonly used lysis buffers. Appl Biosaf. 2017; 22(2):56-59.

9. Swayne D, Beck J. Heat inactivation of avian influenza and Newcastle disease viruses in egg products. Avian Pathol. 2004;33(5): 512-518.

10. King DJ. Evaluation of different methods of inactivation of Newcastle disease virus and avian influenza virus in egg fluids and serum. Avian Dis. 1990;35(3):505-514.

11. Moresco K, Stallknecht D, Swayne D. Evaluation of different embryonating bird eggs and cell cultures for isolation efficiency of avian Influenza A virus and Avian paramyxovirus serotype 1 from real-time reverse transcription polymerase chain reactionpositive wild bird surveillance samples. J Vet Diagn Invest. 2012; 24(3):563-567.

12. Gellis S, Neefe J, Stokes J, Strong L, Janeway C, Scatchard G. Chemical, clinical, and immunological studies on the products of human plasma fractionation. XXXVI. Inactivation of the virus of homologous serum hepatitis in solutions of normal human serum albumin by means of heat. $J$ Clinical Invest. 1948;27(2):239-244.

13. Mitchell S, McCormick J. Physicochemical inactivation of Lassa, Ebola, and Marburg viruses and effect on clinical laboratory analyses. J Clin Microbiol. 1984;20(3):486-489.

14. Nidzworski D, Wasilewska E, Smietanka K, Szewczyk B, Minta Z. Detection and differentiation of Newcastle disease virus and influenza virus by using duplex real-time PCR. Acta Biochim Pol. 2013;60(3):475-480.

15. Thermo Fisher Scientific. Real-time PCR handbook, 2014. https:/www.thermofisher.com/content/dam/LifeTech/global/ Forms/PDF/real-time-pcr-handbook.pdf. Accessed September $15,2017$. 
16. Torchetti MK. Real-time RT-PCR detection of influenza A virus and avian paramyxovirus type-1. 2016. National Veterinary Services Laboratory, SOP-AV-0068.03.

17. Applied Biosystems. MagMAX ${ }^{\mathrm{TM}}-96 \mathrm{AI} / \mathrm{ND}$ Viral RNA isolation kit instruction manual. 1835 M Revision E, 2018. https://www.thermofisher.com/order/catalog/product/AM1835? $\mathrm{SID}=$ srch-hj-AM1835. Accessed September 17, 2017.

18. Safety data sheet $\mathrm{AB} 8500$ : $\mathrm{MagMax}^{\mathrm{TM}}$ Lysis/Binding Solution Concentrate Life Technologies: Carlsbad, CA, 2013. https:// www.thermofisher.com/order/catalog/product/AM8500. Accessed September 17, 2017.
19. Senne D. Virus propagation in embryonating eggs. In Swayne DE, Glisson JR, Jackwood MW, Pearson JE, Reed WM, eds. Laboratory Manual for the Isolation and Identification of Avian Pathogens. 4th ed. Kennet Square, PA: University of Pennsylvania; 1998:235-240

20. Killian M. Hemagglutination assay for avian influenza Virus. In: Spackman E, ed. Avian Influenza Virus. Totowa, NJ: Humana Press; 2008:47-52.

21. Woolcock P. Avian influenza virus isolation and propagation in chicken eggs. In Spackman E, ed. Avian Influenza Virus. Totowa, NJ: Humana Press; 2008:35-46. 